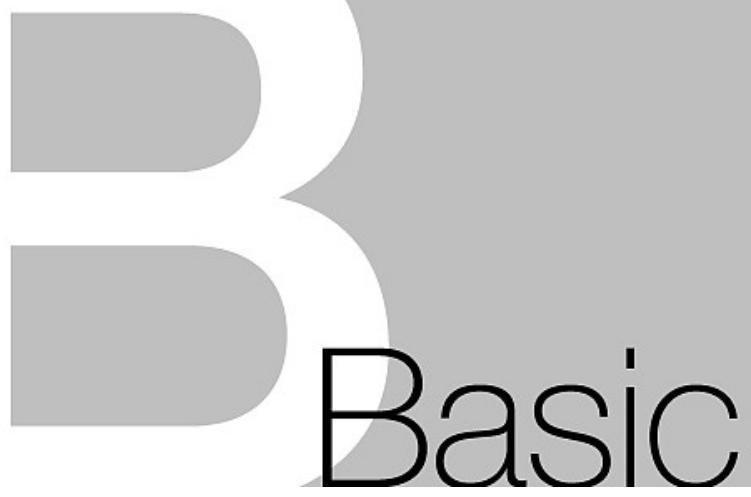

Yong Jiang $^{\mathrm{a}}$, Yueguang Wei ${ }^{\mathrm{b}}$, John. R. Smith ${ }^{\mathrm{a}}$, John W. Hutchinson ${ }^{\mathrm{c}}$, Anthony G. Evans ${ }^{\mathrm{a}, *}$

${ }^{a}$ Materials Department, University of California, Santa Barbara, CA, U.S.A.

${ }^{\mathrm{b}}$ Institute of Mechanics, Chinese Academy of Sciences, Beijing, China

${ }^{\mathrm{c}}$ School of Engineering and Applied Sciences, Harvard University, Cambridge, MA, U.S.A.

\title{
First principles based predictions of the toughness of a metal/oxide interface
}

We describe a first-principles-based strategy to predict the macroscopic toughness of a $\gamma-\mathrm{Ni}(\mathrm{Al}) / \alpha-\mathrm{Al}_{2} \mathrm{O}_{3}$ interface. Density functional theory calculations are used to ascertain energy changes upon displacing the two materials adjacent to the interface, with relaxation conducted over all atoms located within adjoining rows. Traction/displacement curves are obtained from derivatives of the energy. Calculations are performed in mode I (opening), mode II (shear) and at a phase angle of $45^{\circ}$. The shear calculations are conducted for displacements along $<110>$ and $<112>$ of the Ni lattice. A generalized interface potential function is used to characterize the results. Initial fitting to both the shear and normal stress results is required to calibrate the unknowns. Thereafter, consistency is established by using the potential to predict other traction quantities. The potential is incorporated as a traction/displacement function within a cohesive zone model and used to predict the steady-state toughness of the interface. For this purpose, the plasticity of the Ni alloy must be known, including the plasticity length scale. Measurements obtained for a $\gamma-\mathrm{Ni}$ superalloy are used and the toughness predicted over the full range of mode mixity. Additional results for a range of alloys are used to demonstrate the influences of yield strength and length scale.

Keywords: Toughness; Interface; Density functional theory; Multi-scale modeling; Cohesive zone

* Professor Evans passed away on September $9^{\text {th }}, 2009$.

\section{Introduction}

Research on bi-material interfaces has aspired to a computational framework for predicting the engineering adhesion (interface toughness), by starting from basic aspects of atomic bonding [1-4]. While the concept (Fig. 1) is wellestablished, a full (quantitative) implementation has been recent [4]. The elements are as follows.

Step I: A traction-separation relationship for the interface is computed using density functional theory (DFT). This relationship becomes the separation criterion for the interface, implemented as a cohesive zone $(\mathrm{CZ})$.

Step II: The CZ is embedded within an elastic/plastic finite element scheme for calculating the fracture resistance. This step incorporates the multiplicative influence on the fracture resistance of the plastic dissipation occurring in one (or both) of the adjoining materials.

Step III: To generate viable results, the length-scale associated with the plastic deformation must be included. Otherwise, the stress in the cohesive zone never attains the bond rupture stress. The lengthscale is determined from nano-indentation tests conducted on the material immediately adjacent to the interface $[4,5]$.

The influence of mode mixity is fundamental. Among technologically important adhesion challenges, the interface loading ranges from pure mode I (opening) to pure mode II (shear) and many intermediate (mixed mode) combinations $[6,7]$. Consequently, to solve practical problems, the tough- 
Y. Jiang et al.: First principles based predictions of the toughness of a metal/oxide interface
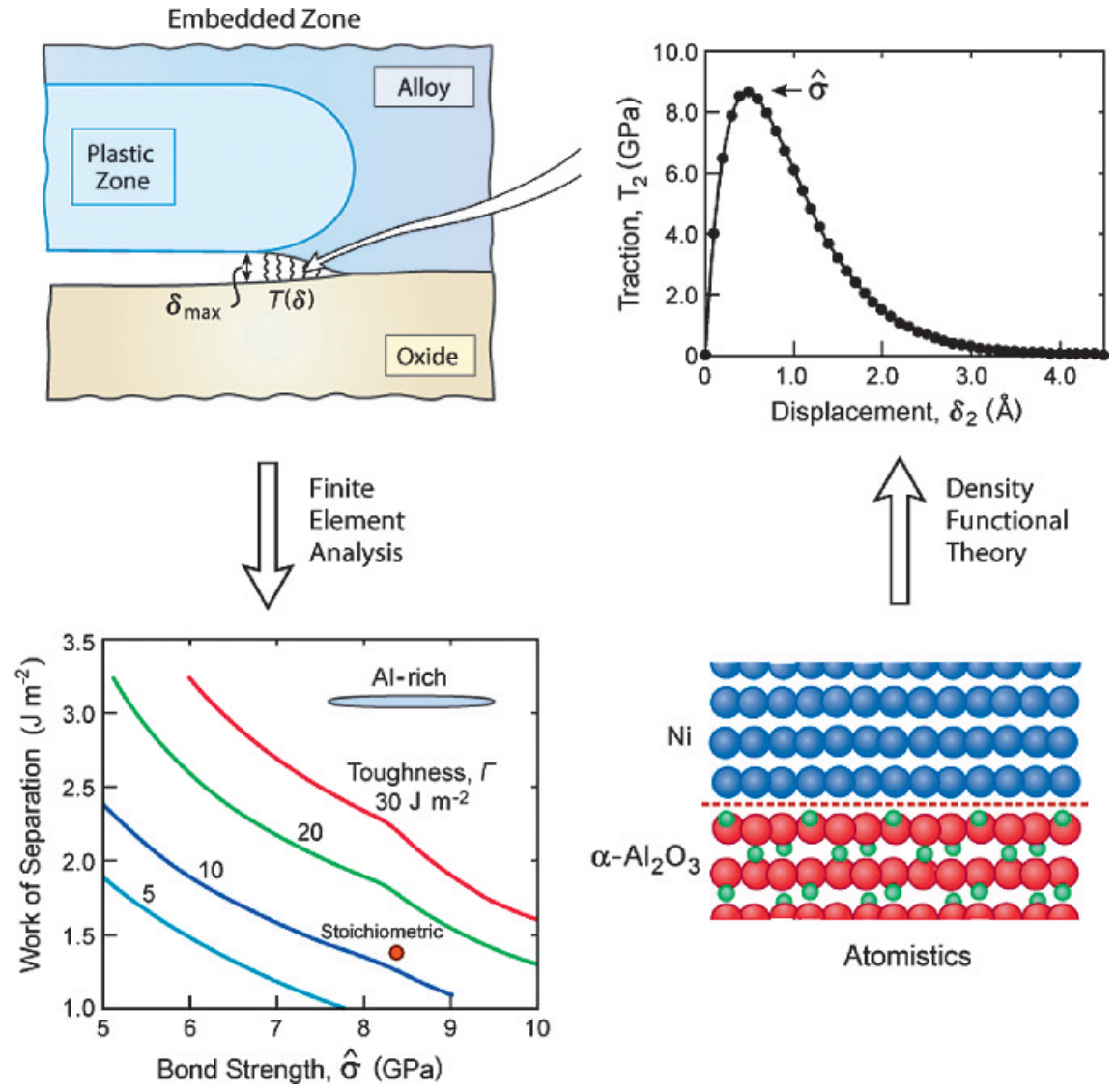

Fig. 1. The concept of using density functional theory calculations of the traction-displacement relation for interface separation as input to a finite element calculation of the interface toughness. For the latter, a plasticity length scale is required to obtain viable solutions.

ness must be characterized over the full range (phase angle $\psi$ between $-90^{\circ}$ and $90^{\circ}$ ). The recent implementation [4] has been conducted for an interface between $\alpha-\mathrm{Al}_{2} \mathrm{O}_{3}$ and $\gamma-\mathrm{Ni}$. The method for relating the tractions to the interface toughness is illustrated in Fig. 1. The present article details the role of density functional theory. Specific results are provided for a stoichiometric interface [8-10]. The methodology whereby the DFT results are used to affirm and validate a generalized potential function for the interface is described. The implementation of this function for the predicting the interface toughness is presented and implications discussed.

\section{The methodology}

The ensuing analysis is presented for the stoichiometric $\gamma$-Ni $\quad(111) \quad(\sqrt{3} \times \sqrt{3}) / \alpha-\mathrm{Al}_{2} \mathrm{O}_{3} \quad(0001) \quad(1 \times 1) \quad$ interface (Fig. 2) [8]. The super-cell used for the calculations includes $8 \mathrm{Ni}$ layers, $12 \mathrm{Al}$ layers and 6 oxygen layers. To determine the total-energy, all possible configurations are assessed for each $\mathrm{Ni}, \mathrm{Al}$, and $\mathrm{O}$ site: symmetry is not enforced. The generalized gradient approximation (GGA) is used for the exchangecorrelation potential. The projector-augmented plane wave method [11] is employed to solve the Kohn-Sham equations. The energy cutoff for the plane wave basis set is taken to be, $E_{\text {cut }}=400 \mathrm{eV}$. To attain equilibrium, relaxations that reduce the total energy are conducted until the net force on each atom falls below a threshold of $20 \mathrm{meV}^{-1}\left(1 \mathrm{eV} \AA^{-1}=1.6 \mathrm{nN}\right)$. In the model (Fig. 3), the upper and lower blocks are displaced rigidly, with two interfacial rows of $\mathrm{Ni}$ atoms permitted to relax, as well as two rows of $\mathrm{O}$ and four rows of $\mathrm{Al}$ atoms. Each calculation is performed by displacing the material blocks in a manner consistent with either opening, shear or mixed mode

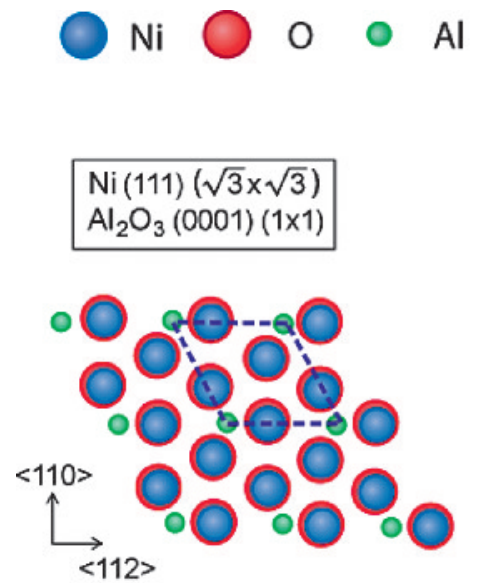

Plan View

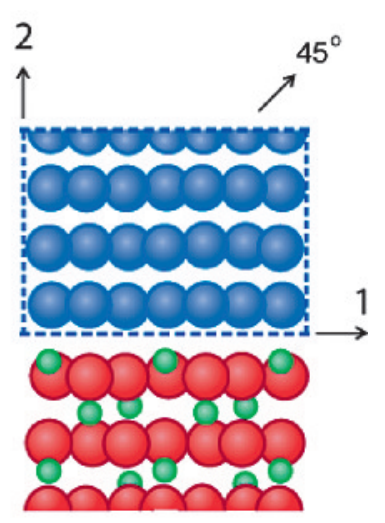

Side View

Fig. 2. The atomic arrangements for the $\mathrm{Ni} / \alpha-\mathrm{Al}_{2} \mathrm{O}_{3}$ interface used for the assessments conducted in this article.

and allowing the rows of atoms on either side of the interface to relax until the net force on each diminishes below the threshold. The calculation is repeated for a sequence of displacements until either the energy difference reduces to zero (opening and mixed mode) or a periodic energy state is obtained (shear). The energy-displacement, $W(\delta)$, curve calculated in this manner is differentiated to obtain the tractions, $T(\delta)$, as illustrated for normal separation on Fig. 1.

To determine the interface toughness from $W(\delta)$, over the full range of mode mixity, a general form for the potential function has been identified, with unknowns to be obtained by fitting to the DFT results for both shear and normal se- 
Y. Jiang et al.: First principles based predictions of the toughness of a metal/oxide interface
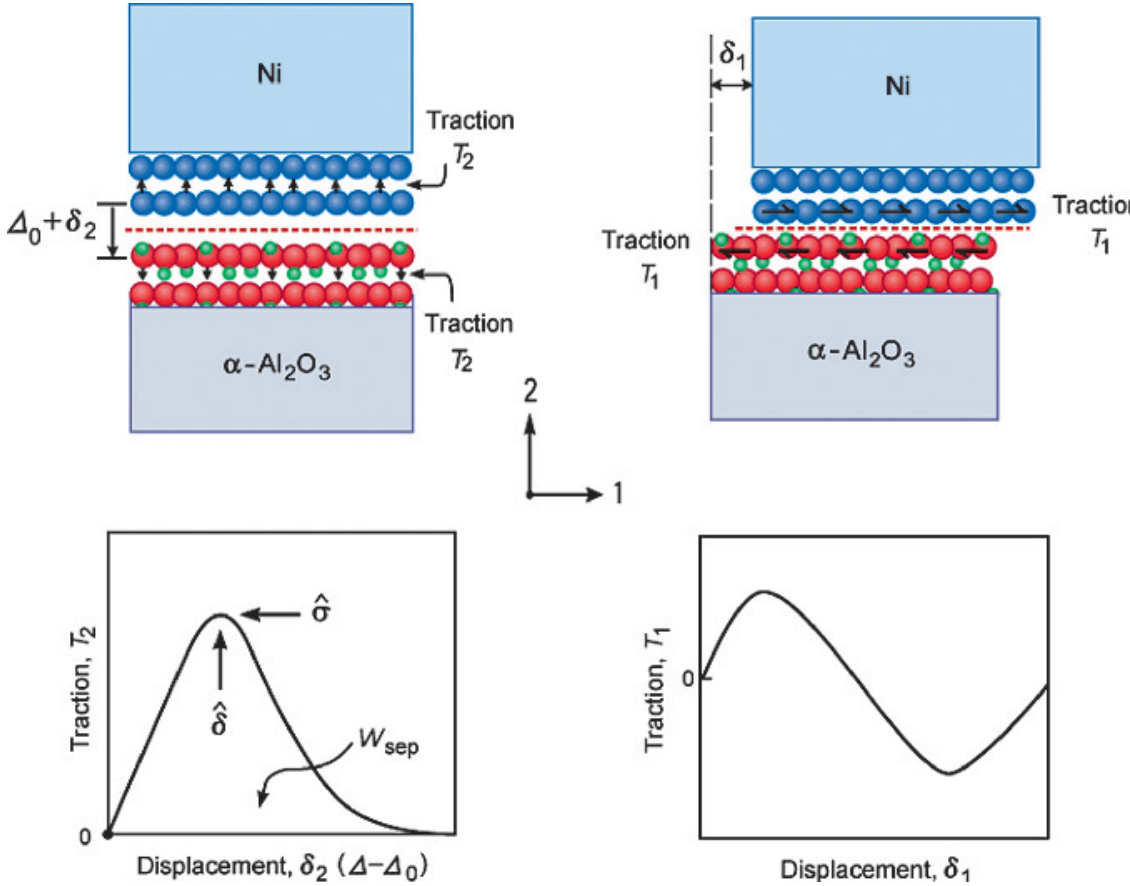

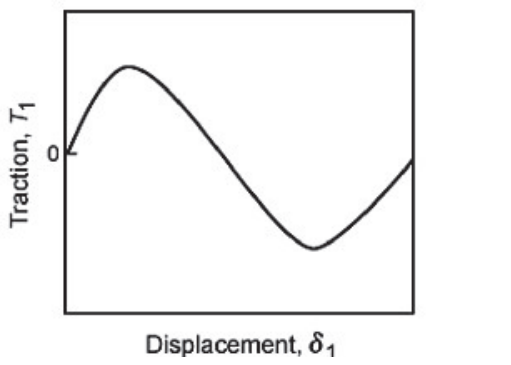

Fig. 3. The model used for calculating the traction-displacement functions. In order to find the energy minimum, the atoms within several rows adjacent to the interface are relaxed in the $(1,2)$ plane until the forces on all atoms drop below $20 \mathrm{meV} \AA$. Outside this domain the $\mathrm{Ni}$ and $\alpha-\mathrm{Al}_{2} \mathrm{O}_{3}$ are displaced rigidly to a specified location and the energy calculated. (a) A typical displacement for normal separation of the interface. (b) Displacements for constrained shear. The equilibrium interface spacing is $\Delta_{0}$. paration. Extended from earlier work $[1-3,12,13]$, the potential function has the form:

$$
\begin{aligned}
W\left(\delta_{1}, \delta_{2}\right)=W_{\text {sep }} & \left\{1-\left(1+\frac{\delta_{2}}{\hat{\delta}}\right) \exp \left(-\frac{\delta_{2}}{\hat{\delta}}\right)\right. \\
+ & \left.f\left(\delta_{1}\right)\left[1+(1+\beta) \frac{\delta_{2}}{\hat{\delta}}\right] \exp \left(-\frac{\delta_{2}}{\hat{\delta}}\right)\right\}
\end{aligned}
$$

Here $\delta_{2}$ is the increase in the normal separation between the bulk Ni and alumina on either side the atomic layers being relaxed (relative to the equilibrium spacing). This definition is consistent with the implementation of displacements when embedded within the cohesive zone, described below. While $\delta_{1}$ is the relative displacement in the 1-direction, parallel to the interface. The displacement at which the normal traction exhibits a maximum is designated $\hat{\delta} ; \beta$ is a dilatation coefficient and $f\left(\delta_{1}\right)$ is the shape-function governing shear. Once $\beta$ and $f\left(\delta_{1}\right)$ have been determined from a few judiciously selected DFT calculations, the tractions derived from (1) can be used as direct input to finite element calculations:

$$
\begin{gathered}
T_{1} \equiv \frac{\partial W}{\partial \delta_{1}}=W_{\text {sep }} \frac{\mathrm{d} f\left(\delta_{1}\right)}{\mathrm{d} \delta_{1}}\left[1+(1+\beta) \frac{\delta_{2}}{\hat{\delta}}\right] \exp \left(-\frac{\delta_{2}}{\hat{\delta}}\right) \\
T_{2} \equiv \frac{\partial W}{\partial \delta_{2}}=\frac{W_{\text {sep }}}{\hat{\delta}}\left[\frac{\delta_{2}}{\hat{\delta}}-\left((1+\beta) \frac{\delta_{2}}{\hat{\delta}}-\beta\right) f\left(\delta_{1}\right)\right] \\
\cdot \exp \left(-\frac{\delta_{2}}{\hat{\delta}}\right)
\end{gathered}
$$

For normal tractions, $T_{2}\left(\delta_{1}=0\right)$, (1) reduces to the "universal" potential [13]. Results have been calculated previously for the stoichiometric interface [8] (Fig. 1): wherein the work of separation is determined from the area under the curve, $W_{\text {sep }}=1.13 \mathrm{~J} \mathrm{~m}^{-2}$, and the bond strength ascertained from the maximum, $\hat{\sigma}=8.3 \mathrm{GPa}$, with the displacement at that stress given by, $\hat{\delta}=0.5 \AA$. The procedure for determining the shear and mixed mode tractions is presented for $\delta_{1}$ displacements along two directions with respect to the Ni lattice (Fig. 2);

(i) Displacements along $<110>$ (the Burgers direction for fcc-Ni), represent the smallest repeat distance, but involve mid-distance interference between atoms across the interface;

(ii) Those along $<112>$ represent a larger repeat distance, but with reduced interference because of the mirror symmetry (evident in the plan view). It remains to ascertain the direction having the lower toughness.

\section{Calculation of the relevant tractions}

Before proceeding, context governing the cohesive zone parameters to be used in toughness determination and the total number of atomic layers, $N$, used in the DFT relaxation procedure is provided by the following considerations.

(i) The work of separation, $W_{\text {sep }}$, and the maximum stress for normal separation, $\hat{\sigma}$, are independent of $N$, provided that enough layers have been taken to fully capture relaxation effects.

(ii) Full interface potentials independent of $N$ can be ascertained by subtracting the small elastic displacements $[14,15]$.

(iii) For cohesive zones embedded within plastically deforming solids $[2,16] W_{\text {sep }}$, as well as the peak stresses for normal separation, $\hat{\sigma}$, and shear, are the three parameters having the primary influence on the macroscopic toughness.

Details related to the specific functional shape of the separation behavior have a relatively weak influence. As a consequence of these three features, the potential from the entire atomic multi-layer has been chosen to characterize the cohesive zone, without subtracting the elastic displacements.

The role of the constraints used in the DFT calculations also merits discussion. The constraint boundary conditions at a crack are controlled by local stress-strain fields. In 
Y. Jiang et al.: First principles based predictions of the toughness of a metal/oxide interface

turn, these depend on the interface characteristics. In the computational model of the toughness [4], the potential adjusts to the actual local constraints. Namely, given an accurate representation of interface separation, the models accommodate any combination of local constraints. An example is presented in Section 5 for a mode II interface crack. The task assigned to DFT is thus to identify the shape function $f\left(\delta_{1}\right)$ and the dilatation parameter $\beta$ and to establish that the potential is accurate for the full range of possible constraints. The constraints used are not intended to reflect those involved in interface crack extension.

To establish consistency, and to provide the highest fidelity, two limiting boundary conditions are employed to compute $f\left(\delta_{1}\right)$ and $\beta$ :

(i) constrained shear with $\delta_{2}=0$ and

(ii) unconstrained shear with $T_{2}=0$.

Constrained shear results are obtained by enforcing a series of small displacements, $\delta_{1}$, along the shear direction 1, relaxing all of the atoms around the interface, and then determining the energy. A transverse compression is induced (Fig. 3b). The magnitude of this compression is calculated by perturbing through a small displacement in the 2-direction, $\mathrm{d} \delta_{2}$, relaxing again, and re-computing the energy. Upon repeating for several displacements, the derivative of this energy with $\mathrm{d} \delta_{2}$ gives the traction $T_{2}$ at the designated $\delta_{1}$. The compressions induced are expected to be large in magnitude because all of the elasticity is confined to the few atomic layers over which the DFT calculations are conducted (the outer blocks are rigid). The situation is closely analogous to the stresses induced around an array of dislocations [17]. Un- constrained shear results are obtained by imposing a displacement in the 1-direction $\delta_{1}$, computing the energy change at that $\delta_{1}$ upon imposing small displacements in the 2-direction, $\mathrm{d} \delta_{2}$, until a location is found where the net $T_{2}$ traction is zero. This process is repeated for each $\delta_{1}$ until a full energy-displacement curve has been generated. Recall that, in all calculations, the near-interface layers are allowed to relax fully in response to the imposed displacements, until the force on each atom converges to $<30 \mathrm{nN}$. Similar DFT methods have been used to examine shear phenomena in other systems [18, 19]. The energetics involved are plotted on Fig. 4, with respect to the equilibrium state. Note that for both the $<112>$ and $<110>$ directions, the two different constraints have similar effects on total energies, largely due to local relaxation at the interface.

To illustrate the importance of relaxation, calculations for an un-relaxed shear are superimposed on Fig. 4. The large barriers (at $\delta_{1}=1.35 \AA$ along $<110>$ and $3.0 \AA$ along $<112>$ ) reflect unfavorable atomic arrangements across the interface and consequently, a significant increase in total energy. At those unfavorable configurations, evident in the plan views of the atom positions (Fig. 4), the outmost $\mathrm{Ni}$ atoms reside at hollow or bridge sites displaced from their most favorable locations above the $\mathrm{O}$ atoms of the $\alpha-\mathrm{Al}_{2} \mathrm{O}_{3}[8-10]$. The atomic rearrangements enabled by local relaxation effectively mitigate these barriers.

The derivative of the total energies gives the tractions, $T_{1}$. Those for constrained shear along $<112>$ are plotted on Fig. 5a. The Burgers vector is, $b_{112}=4.54 \AA$, and the peak stress, $\hat{T}_{1}= \pm 7 \mathrm{GPa}$ at $\delta=1.0 \AA$. Note that the curve lacks

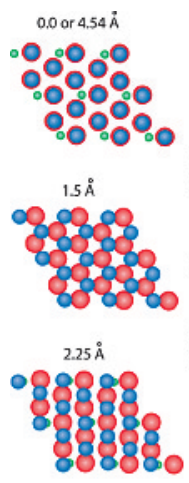

(a)
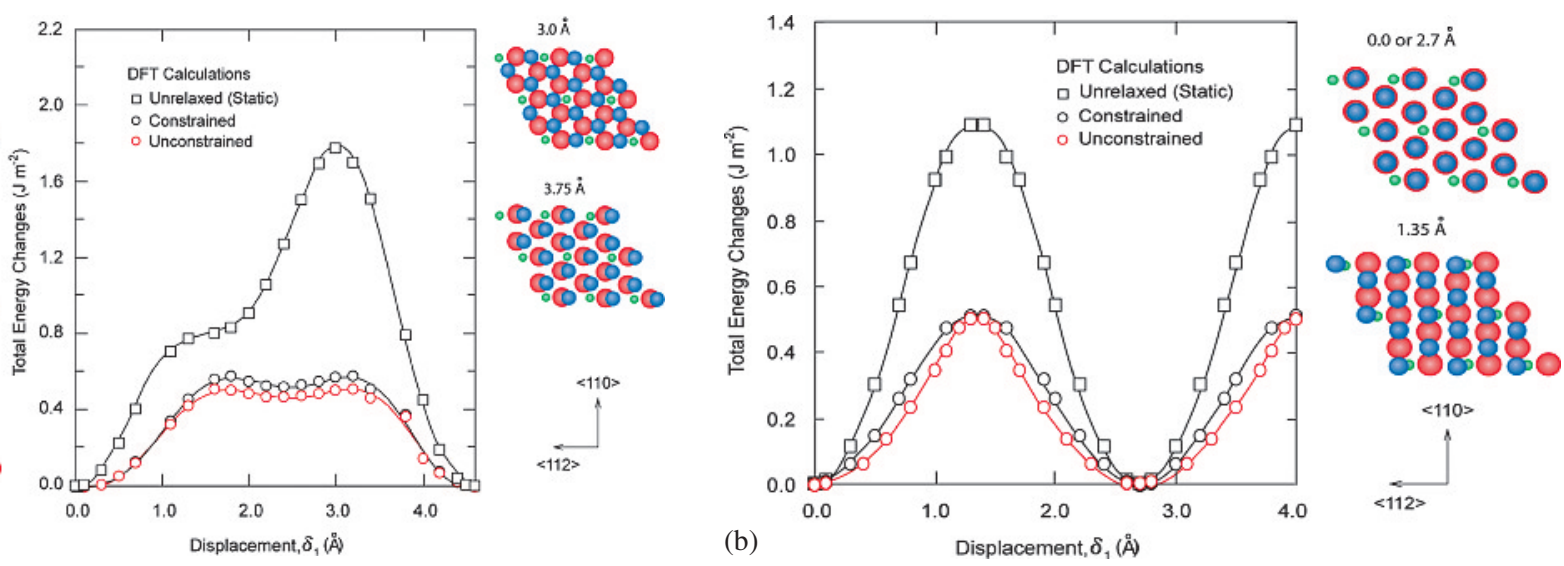

Fig. 4. The total energy changes under different constraint conditions. Plan views of the interface region show the interference between atoms. (a) Upon displacing along $<112>$. (b) Displacements along $<110>$.

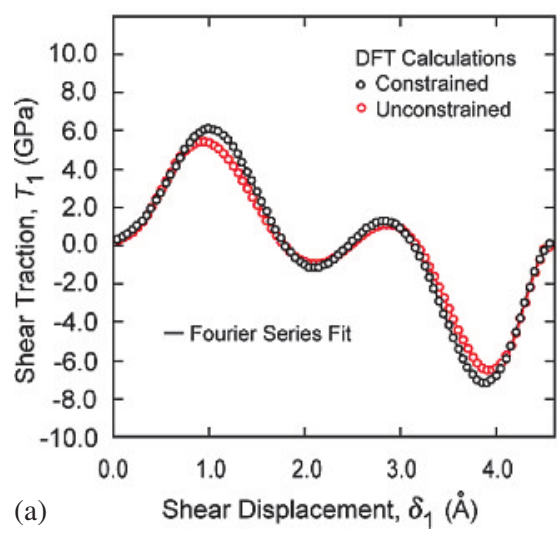

4

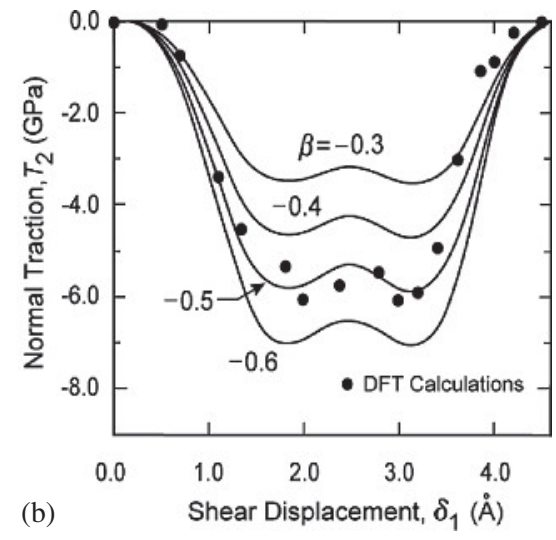

Fig. 5. The (a) shear and (b) normal tractions calculated for constrained shear along $<112>$. The corresponding results for unconstrained shear are included in (a) for the shear tractions. The fit to the shear tractions to obtain $f\left(\delta_{1}\right)$ is shown in (a). The predictions made using this fit and $\beta=-0.5$ shown in: (a) for unconstrained shear and (b) the normal stress induced by constrained shear. 


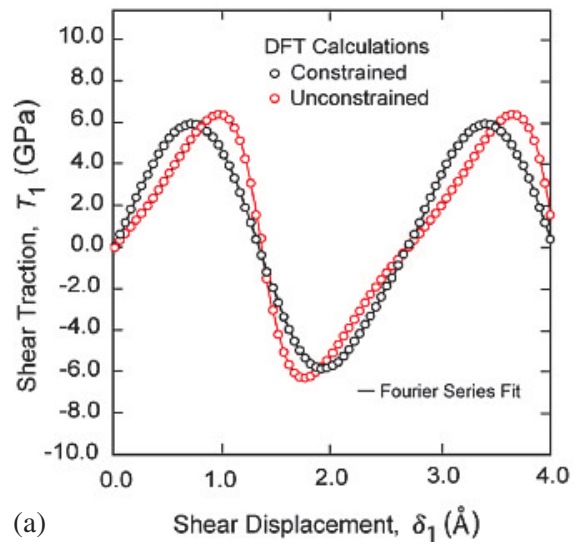

symmetry about zero, because of the asymmetric arrangement of the $\mathrm{Ni}$ (relative to $\mathrm{Al}$ ) atoms. The corresponding results along $<110\rangle$ are plotted on Fig. 6a. The repeat displacement is now, $b_{110}=2.7 \AA$, and the peak stress, $\hat{T}_{1}= \pm 6.4 \mathrm{GPa}$ at $\delta_{1}=1 \AA$. Symmetry is retained. The induced compressive stresses, $T_{2}$, calculated for shear along $<110>$ are plotted on Fig. $5 b$ and those along $<112>$ on Fig. 6b. Note that these stresses are substantial and comparable in magnitude to the shear tractions, as in the analogous situation for an array of dislocations [17]. The corresponding $T_{1}$ tractions calculated for unconstrained shear are superimposed on Figs. 5a and 6a. Note that the absence of constraint changes the peak traction by only about $5 \%$, consistent with the small differences between constrained and unconstrained total energies (Fig. 4).

The similarity between the constrained and unconstrained total energies and shear tractions (Figs. 4a, b, 5a, and 6a) implies that the toughness predictions are insensitive to the specific constraints that develop on the cohesive zone, enabling us to proceed with the knowledge that our method is robust.

A consistency-check is provided by conducting calculations for displacements along a $45^{\circ}$ orientation with respect to the interface (Fig. 7). For this purpose, the blocks are displaced by $\delta_{1}=\delta_{2}=\delta_{45} / \sqrt{2}$ and the atoms in the interfacial layer relaxed along the 1 and 2 directions until the net force on each again converges to the threshold. The corresponding energy, $W_{45}\left(\delta_{45}\right)$, is computed. The derivative gives the net traction along the $45^{\circ}$ direction, $T_{45}\left(\delta_{45}\right)$. The results for in-plane displacements along $<112>$ are plotted on Fig. 7 (red dots). Note that the work of separation (the area under the curve) is precisely the same as that obtained under normal separation (Fig. 1), because no interface dislocations have been generated nor atomic positions interchanged during the separation process.

\section{The potential function}

The DFT results from either of the two limiting boundary conditions could be used to determine the two unknowns in the potential function, $f\left(\delta_{1}\right)$ and $\beta$. For constrained shear, (1) and (2) give

$$
\begin{aligned}
& W=W_{\text {sep }} f\left(\delta_{1}\right), \quad T_{1}=W_{\text {sep }} \frac{\mathrm{d} f\left(\delta_{1}\right)}{\mathrm{d} \delta_{1}}, \\
& T_{2}=\beta \frac{W_{\text {sep }}}{\hat{\delta}} f\left(\delta_{1}\right)
\end{aligned}
$$

Thus, $f\left(\delta_{1}\right)$ is obtained directly from $T_{1}\left(\delta_{1}\right)$, while $\beta$ is determined from a best fit to $T_{2}\left(\delta_{1}\right)$. Using $T_{1}\left(\delta_{1}\right)$ from Figs. 5a and 6a, along with $W_{\text {sep }}$ from Fig. 1, a Fourier series has been used to represent $f\left(\delta_{1}\right)$. To obtain the fits presented on Figs. 5 and 6 , five series terms were used for shear along $<110>$; while, due to the secondary peak, nine terms were required along $<112>$. Following determination of $f\left(\delta_{1}\right)$, the normal stresses $T_{2}\left(\delta_{1}\right)$ have been calculated from (3) for a range of
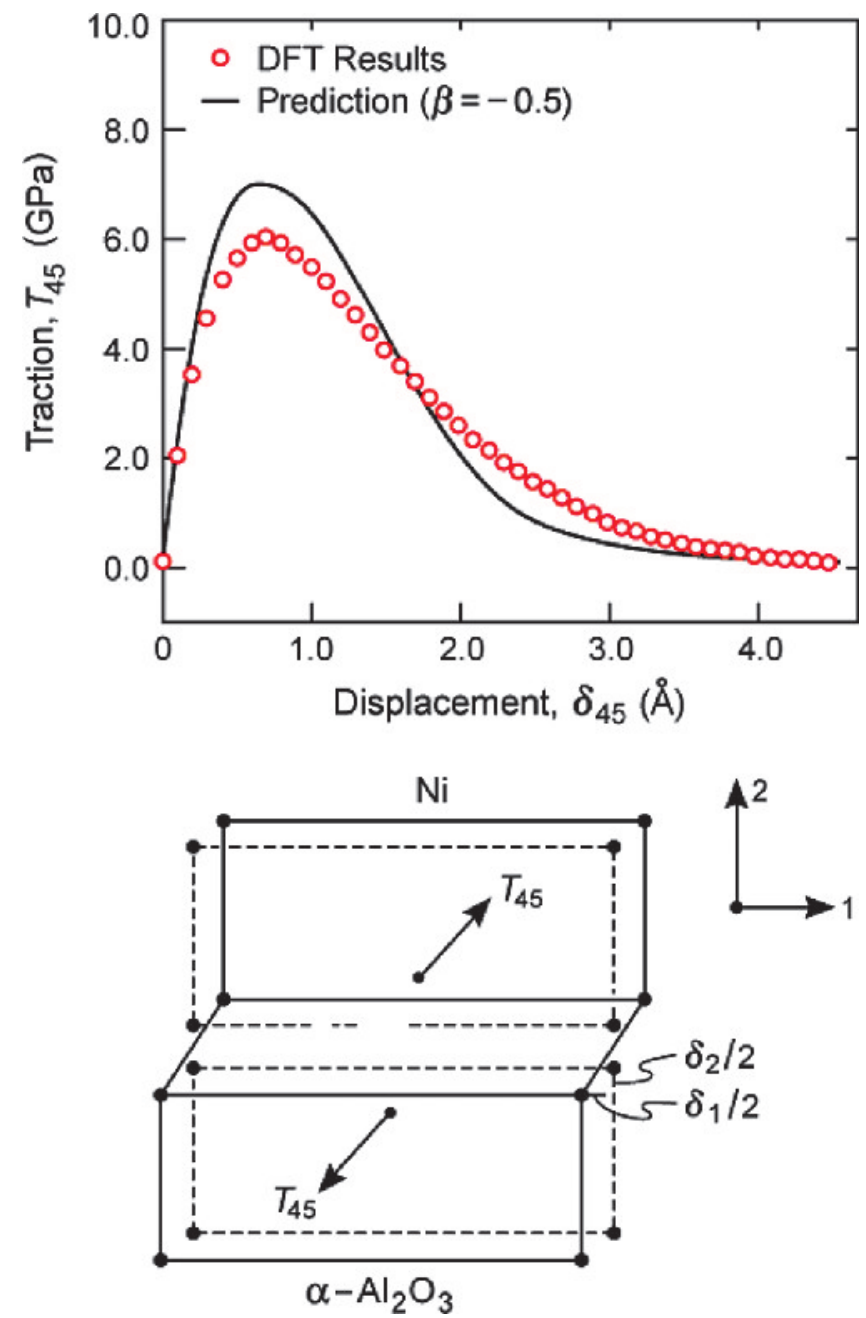

Fig. 7. The DFT calculations for mixed mode displacements at $45^{\circ}$ with shear along $<112>$. Also shown are the predictions made using the same $f\left(\delta_{1}\right)$, again with $\beta=-0.5$. 
Y. Jiang et al.: First principles based predictions of the toughness of a metal/oxide interface

possible values of $\beta$ (Figs. 5b and $6 \mathrm{~b}$ ). For both directions, a best fit to the DFT results is found for $\beta \approx-0.5$. Equivalent determinations could be obtained using the unconstrained shear results, wherein $T_{2}=0$ implies

$\frac{\delta_{2}}{\hat{\delta}}=-\frac{\beta f\left(\delta_{1}\right)}{1-(1+\beta) f\left(\delta_{1}\right)}$

such that $f\left(\delta_{1}\right)$ and $\beta$ can be obtained by simultaneously fitting either $W\left(\delta_{1}\right)$ in Eq. (1) or $T_{1}\left(\delta_{1}\right)$ in Eq. (2a) and $\delta_{2}\left(\delta_{1}\right)$ in Eq. (4).

With both unknowns now determined, predictions from the potential function of Eq. 3 with $\beta=-0.5$ can be compared with other DFT results. As one check, the potential has been used to reproduce the DFT unconstrained shear results in Figs. 5a and 6a. For another, the tractions caused by the $45^{\circ}$ displacements have been calculated using (Fig. 7):

$\mathrm{d} W=T_{1} \mathrm{~d} \delta_{1}+T_{2} \mathrm{~d} \delta_{2}=T_{45^{\circ}} \mathrm{d} \delta_{45^{\circ}}$

with $\delta_{45^{\circ}}=\sqrt{2} \delta_{1},\left(\delta_{1}=\delta_{2}\right)$. A small discrepancy exists between the DFT results and those predicted by the potential. It remains to determine the source of the discrepancy. Nevertheless, the ability of the potential Eq. (1) to adequately characterize such a wide range of separation characteristics provides confidence for its use within a cohesive zone in the ensuing toughness model.

\section{The interface toughness}

The preceding traction relations enter into a finite element scheme for predicting the interface toughness [4]. The procedure is demonstrated for a system of practical interest involving the $\mathrm{Ni}(\mathrm{Al}, \mathrm{Cr}, \mathrm{Co}, \mathrm{Y})$ bond coat alloys used for the oxidation protection of Ni-superalloys. Upon $\alpha-\mathrm{Al}_{2} \mathrm{O}_{3}$ formation during high temperature exposure, the alloy closest to the interface becomes $\mathrm{Al}$ depleted and develops a composition in the $\gamma$-phase field [20]. The properties of the alloy in this field are most pertinent to the assessment of the adhesion. Moreover, loss of adhesion (when it happens) typically occurs after cooling [7], so the properties at ambient are relevant.

The properties pertinent to a commercial alloy are [5]: Young's modulus, $E_{\mathrm{Ni}}=170 \mathrm{GPa}$, Poisson ratio, $v=0.3$, yield strength, $\sigma_{\mathrm{Y}}=700 \mathrm{MPa}$, coefficient for power law strain hardening, $N=0.2$. The plasticity length scale has been estimated from nano-indentation measurements as, $\ell=50 \mathrm{~nm}$ [21]. The reader is referred to Wei and Hutchinson $[4,22]$ for a detailed description of the length-scale and how it enters into the constitutive representation for the plasticity used in the toughness model. The plastic zone size in the $\mathrm{Ni}$ alloy is scaled by a second length parameter

$R_{0}=\frac{1}{3 \pi\left(1-v^{2}\right)} \frac{E_{\mathrm{Ni}} W_{\mathrm{sep}}}{\sigma_{\mathrm{Y}}^{2}}$

For the stoichiometric interface, $R_{0}=46 \mathrm{~nm}, \hat{\sigma} / \sigma_{\mathrm{Y}}=11.9$ and $l / R_{0} \cong 1$ [4]. For this limited set of conditions, the macroscopic interface toughness, $\Gamma_{\mathrm{ss}}$, has the following functional dependence $[3,4,18]$ :

$\Gamma_{\mathrm{ss}}=W_{\mathrm{sep}} F\left(\frac{\hat{\sigma}}{\sigma_{\mathrm{Y}}}, \frac{\ell}{R_{0}}, \psi\right)$ where $F$ is a dimensionless function capturing the toughness enhancement above the atomistic work of separation. The toughness model is formulated for steady-state crack growth, and $\Gamma_{\mathrm{ss}}$ is the associated critical energy release rate $[3,4,22]$. The toughness envelopes calculated for displacements $<112>$ and $<110>$ are plotted on Fig. 8a. The small difference between the two orientations under nominal

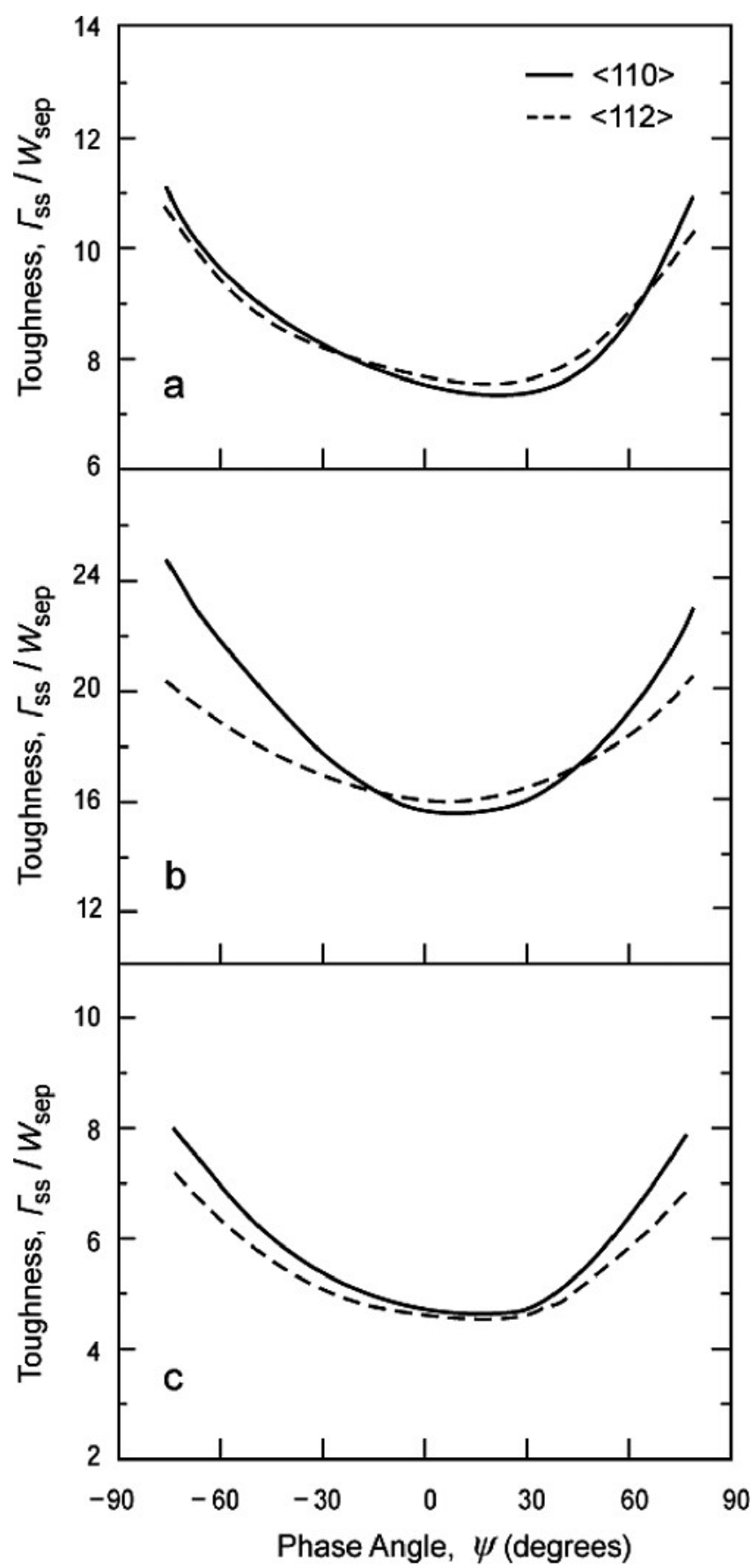

Fig. 8. Macroscopic mixed mode toughness for the stoichiometric $\gamma-\mathrm{Ni} / \alpha-\mathrm{Al}_{2} \mathrm{O}_{3}$ interface. The parameters characterizing the interface potential have been determined from DFT calculations described in the text. Elasticity parameters are also specified in the text. (a) The parameters specifying the plasticity of the $\mathrm{Ni}$ alloy are $\sigma_{\mathrm{Y}}=700 \mathrm{MPa}$, $R_{0}=46 \mathrm{~nm}, \ell=46 \mathrm{~nm}\left(\ell / R_{0}=1\right)$. (b) The effect of decreasing the Ni alloy yield strength from $\sigma_{\mathrm{Y}}=700 \mathrm{MPa}$ to $600 \mathrm{MPa}$. (c) The effect of increasing the plasticity length parameter for the $\mathrm{Ni}$ alloy from $\ell=46 \mathrm{~nm}$ to $92 \mathrm{~nm}$.

Int. J. Mat. Res. (formerly Z. Metallkd.) 101 (2010) 1 
Y. Jiang et al.: First principles based predictions of the toughness of a metal/oxide interface

mode $\mathrm{I}\left(\psi=0^{\circ}\right)$ is attributed to the elastic asymmetry that creates some plastic shearing of the $\mathrm{Ni}$ at the tip: that is, the local conditions are not strictly mode I [6]. For the same reason, the mixed mode toughness curves are not symmetric about $\psi=0^{\circ}$. The multiplicative enhancement of the toughness above $W_{\text {sep }}$ is due to the plastic dissipation in the Ni alloy. In the presence of a significant mode II component, the toughness is further amplified, because additional plasticity is induced in the Ni by the extra shear. The similarity in toughness between the two directions, <112> and $\langle 110\rangle$, reflects the closeness in the shearing curves before the peak (Figs. 5a and 6a), with the understanding that the tractions are dominated by the exponential term, $\exp \left(-\delta_{2} / \hat{\delta}\right)$, in (2): namely, the "tail" of the shearing input, $f\left(\delta_{1}\right)$, beyond the peak, has little influence.

The nature of the constraints has been examined by calculating the normal displacements, as a function of the distance from the crack front. An example for a near-mode II crack (phase angle, $\psi=86^{\circ}$ extending along $\langle 110\rangle$ is presented on Fig. 9. Note that the crack is open up to the end of the cohesive zone so that there is no crack face friction. The tractions on the cohesive zone thus comprise shear with a small normal tension. The result is essentially the same for extension along $\langle 112\rangle$. This stress state causes the toughness predictions for this interface to be insensitive to the magnitude of $\beta$ [4].

Two additional sets of simulations have been used to highlight the roles of plasticity and of the interface strength, $\hat{\sigma}$. Results presented in Fig. 8b have been computed with precisely the same physical parameters except that the yield strength of the Ni has been decreased by $15 \%$ from 700 to $600 \mathrm{MPa}$. This small reduction doubles the toughness over the entire range of mixity. The primary factor enhancing the toughness is the increase in $\hat{\sigma} / \sigma_{\mathrm{Y}}$ (from 11.9 to 13.8) even though $\ell / R_{0}$ also decreases (from 1 to 0.73 ), [4]. This example emphasizes that, despite the direct scaling of the toughness with the work of separation, the interface strength is the dominant parameter, through its role in determining the plastic dissipation.

The effect of changing only the plasticity length parameter, from $46 \mathrm{~nm}$ to $92 \mathrm{~nm}$ (such that $\ell / R_{0}$ is doubled), with $\hat{\sigma} / \sigma_{Y}$ unchanged, is presented on Fig. 8c. This increase in

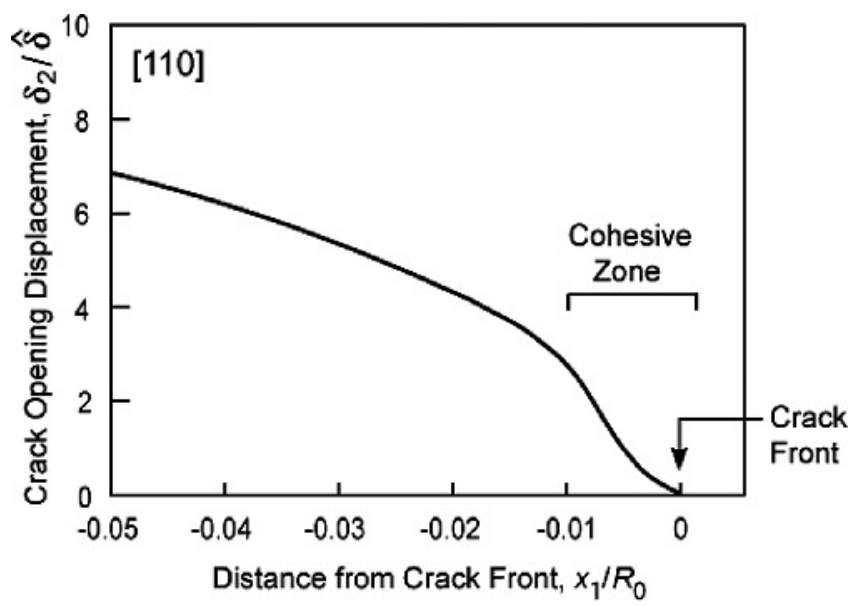

Fig. 9. The profile for an interface crack extending along [110] at a phase angle, $\psi=86^{\circ}$. For this example the crack faces are not in contact, such that shear and tensile tractions are present at the cohesive zone. length scale causes a $30 \%$ reduction in the toughness. This parameter in the plasticity theory controls the stress elevation near the crack tip due to local plastic strain gradients. The larger $\ell$, the higher the crack tip stresses, and the more readily the interface separation conditions are met. Lower toughness is the consequence. These trends further emphasize the important role of the interface strength, since local stresses acting on the interface must attain levels set by $\hat{\sigma}$, otherwise the crack cannot propagate.

\section{Concluding comment}

The preceding assessment has highlighted the use of the interface potential function as an effective way to incorporate first principles results for the separation of interfaces into computational models for toughness. The potential must be calibrated by using a combination of DFT results obtained for normal separation and for shearing displacements. The latter can be tested by using either constrained or unconstrained shear conditions to represent all possible levels of local stresses on the cohesive zone. It was found that the DFT shear results critical to toughness computations were insensitive to such constraints, an indication of robustness of the method. For viability, it must be demonstrated that the potential is accurate for a full range of mixed mode atomistic simulations, as illustrated by the potential invoked in this article, which captures all features relevant to the macroscopic toughness model.

The authors are grateful for Air Force Office of Scientific Research (AFOSR) support from Grant No. FA9550-05-C-0039. One of us (JRS) would like to thank the ONR (Grant N00014-08-1-1164) for partial support.

\section{References}

[1] Y. Sun, G.E. Beltz, J.R. Rice: Mater. Sci. Eng. A 67 (1993) 170. DOI:

[2] V. Tvergaard, J.W. Hutchinson: J. Mech Phys Solids 41 (1993) 1119. DOI:

[3] Y. Wei, J.W. Hutchinson: Int. J. Fracture 95 (1999) 1. DOI:

[4] Y. Wei, J.W. Hutchinson: Philos. Mag. 88 (2008) 3841. DOI:

[5] K.J. Hemker, B.G. Mendis, C. Eberl: Mater. Sci. Eng. A 483-484 (2008) 727. DOI:

[6] J.W. Hutchinson, Z. Suo: Adv. Appl. Mech., 29 (1992) 63. DOI:

[7] A.G. Evans, J.W. Hutchinson: Surface \& Coatings Technology, 201 (2007) 7905. DOI:

[8] J.R. Smith, Y. Jiang, A.G. Evans: Int. J. Mat. Res. 98 (2007) 1214. DOI:

[9] W. Zhang, J.R. Smith, A.G. Evans: Acta Mater 50 (2002) 3803. DOI:

[10] Y. Jiang, J.R. Smith, A.G. Evans: Appl. Phys. Lett. 92 (2008) 141918. DOI:

[11] P.E. Blöchl: Phys. Rev. B 50 (1994) 17953. DOI:

[12] A. Needleman: J. Mech. Phys. Solids 38 (1990) 289. DOI:

[13] J.H. Rose, J. Ferrante, J.R. Smith: Phys. Rev. Lett. 47 (1981) 675. DOI:

[14] R.L. Hayes, M. Ortiz, E.A. Carter: Phys. Rev. B 69 (2004) 172104. DOI:

[15] A. Van der Ven, G. Ceder: Acta Materialia. 52 (2004) 1223. DOI:

[16] V. Tvergaard, J.W. Hutchinson: J. Mech. Phys. Solids 40 (1992) 1377. DOI:

[17] J.P. Hirth, J. Loethe: Theory of Dislocations, McGraw-Hill, NY (1968). DOI: 
Y. Jiang et al.: First principles based predictions of the toughness of a metal/oxide interface

[18] S. Ogata, J. Li, S. Yip: Science 298 (2002) 807. DOI:

[19] T. Liang, W. Gregory Sawyer, S.S. Perry, S.B. Sinnott, S.R. Phillpot: Phys. Rev. B 77 (2008) 104105. DOI:

[20] T. Xu, S. Faulhaber, C. Mercer, M. Maloney, A. Evans: Acta Materialia 52 (2004) 1439. DOI:

[21] K.J. Hemker: private communication (2008).

[22] Y. Wei, J.W. Hutchinson: J. Mech. Phys. Solids 51 (2003) 2037. DOI:

(Received April 7, 2009; accepted October 21, 2009)

\section{Bibliography}

DOI 10.3139/146.110254

Int. J. Mat. Res. (formerly Z. Metallkd.)

101 (2010) 1; page $1-8$

(C) Carl Hanser Verlag GmbH \& Co. KG

ISSN 1862-5282

\section{Correspondence address}

Prof. John Smith

Materials Department, University of California, Santa Barbara

1355 B Engineering II, University of California, Santa Barbara

California, 93106-5050, U.S.A

Tel.: +12484961874

Fax: +12486426219

E-mail: johnjrs@engr.ucsb.edu
You will find the article and additional material by entering the document number MK110254 on our website at www.ijmr.de 